\title{
A Fatal Case of Neuroleptic Malignant Syndrome After Paralytic Bowel in a Patient Taking Antiparkinson Medication
}

\author{
Domenico Campa ${ }^{1}$, Manuela Ariello ${ }^{1}$, Rosaria Castaldo ${ }^{1}$, Francesco Zibella ${ }^{1}, \underline{\text { Paolo Ferrazza }}^{2}$, Salvatore Del Gaudio ${ }^{1}$ \\ ${ }^{1}$ Department of Emergency Medicine, Pineta Grande Hospital, Caserta, Italy
}

${ }^{2}$ LN Age, Roma, Italy

Received: 29/12/2015

Accepted: 08/02/2016

Published: $18 / 05 / 2016$

How to cite this article: Campa D, Ariello M, Castaldo R, Zibella F, Ferrazza P, Del Gaudio S, A fatal case of neuroleptic malignant syndrome after paralytic bowel in a patient taking antiparkinson medication. EJCRIM 2016;3:doi:10.12890/2016_000368

Conflicts of Interests: The Authors declare that there are no competing interests.

Acknownledgements: The Authors would like to thank Dr. Capria Francesco and Dr. Melengu Ergena for help with scientific writing.

This article is licensed under a Commons Attribution Non-Commercial 4.0 License

\section{ABSTRACT}

Objectives: We report a fatal case of neuroleptic malignant-like syndrome, which occurred as a consequence of paralytic bowel in a 72-yearold woman on treatment with antiparkinson medication.

Case description: Contrast enhanced computerized tomography of the chest and abdomen demonstrated the presence of paralytic bowel. Results: The patient died.

Conclusions: Physicians involved in the treatment of patients affected by Parkinson's disease should take into consideration the possibility of dopaminergic drug malabsorption due to paralytic bowel as a possible cause of neuroleptic malignant-like syndrome.

\section{LEARNING POINTS}

- Clinical features of neuroleptic malignant-like syndrome (NMLS) in parkinsonian patients are similar to those of neuroleptic malignant syndrome (NMS), which is a potentially fatal condition associated with the withdrawal of antipsychotic medication.

- Paralytic bowel is a condition frequently impairing medication absorption in the absence of modifications to the normal daily intake of antiparkinson drugs.

- The present case report describes the fatal outcome of NMLS following paralytic bowel in a parkinsonian patient on levodopa and amantadine therapy.

\section{KEYWORDS}

Neuroleptic malignant-like syndrome (NMLS); Paralytic bowel (PB); Parkinson's disease (PD).

\section{INTRODUCTION}

Neuroleptic malignant syndrome (NMS) is an idiosyncratic, rare and potentially fatal condition frequently associated with the withdrawal of antipsychotic medication ${ }^{[1]}$. The following symptoms are often described: persistent high temperature, stupor, autonomic and mental dysfunction, and rhabdomyolysis. Neuroleptic malignant-like syndrome (NMLS) is a clinically similar condition associated with sudden withdrawal of or abrupt reduction in dopamine agonists ${ }^{[2]}$.

Paralytic bowel (PB) is a condition which frequently impairs medication absorption in the absence of modifications to the normal daily intake. NMLS caused by PB has not been widely described in the literature, although there is a relationship between NMS and gastroparesis. 
The present report describes a case of NMLS in a parkinsonian patient on therapy with levodopa and amantadine who, due to the concomitant development of PB, experienced drug malabsorption leading to substantial withdrawal of dopamine agonist therapy.

\section{CASE DESCRIPTION}

A parkinsonian female patient aged 72 and bedridden for non-union of a femoral fracture, presented to the emergency department of our hospital with a 4-day history of high temperature $\left(>39.5^{\circ} \mathrm{C}\right)$ and diffuse abdominal pain, distention and constipation without intestinal sounds. The patient's relatives reported normal intake of her daily antiparkinson medication, consisting of levodopa and amantadine. At admission, the clinical picture was as follows: hyperpyrexia (axillary temperature $39.8^{\circ} \mathrm{C}$ ), mild sinus tachycardia (112 bpm), blood pressure of $178 / 93 \mathrm{mmHg}$, oxygen saturation $\left(\mathrm{SaO}_{2}\right)$ on pulse oximetry of $93 \%$, and respiratory rate of 20 . Abdominal bloating was noted on physical examination and mixed alkalosis was confirmed by arterial blood gas (ABG) analysis. Laboratory tests demonstrated the following abnormalities: increased total creatine phosphokinase (CPK) up to $614 \mathrm{mlU} / \mathrm{ml}$, increased lactate dehydrogenase (LDH) up to 1034 IU/I, increased myoglobin up to $142 \mathrm{ng} / \mathrm{ml}$, severe hypokalemia with serum potassium concentrations of $2.6 \mathrm{mEq} / \mathrm{l}$, and an elevated white blood cell count (WBC) of 12,000 .

In the following days, urine culture, viral tests and multiple blood cultures showed negative results, while chest and abdominal X-rays could not be used for diagnostic purposes as our patient could only rest in a dorsal recumbent position which made it hard to perform these investigations properly. Ceftriaxone prophylaxis ( $1 \mathrm{~g}$ twice a day) and fluid therapy were started and normal intake of the medications taken at home was maintained. However, the patient showed no improvement. Her high temperature persisted and was resistant to nonsteroidal anti-inflammatory drugs (NSAIDs) and corticosteroids. After a further 4 days, the patient's general condition worsened, and was accompanied by decreased diuresis and rhabdomyolysis confirmed by laboratory tests. Despite the renal impairment and rhabdomyolysis, a contrast enhanced computerized tomography (CT) scan of the chest and abdomen was performed after adequate hydration in order to exclude surgical pathology. The CT scan confirmed the presence of PB, with the consequent functional intestinal occlusion responsible for the malabsorption of antiparkinson drugs. Usually, a clinical examination together with an X-ray (orthostatic and clinostatic) of the abdomen or an abdominal echography is sufficient for the diagnosis of PB. However, in this case it was decided to proceed with the CT scan of the abdomen because it could be carried out more easily in the supine decubitus position and also because, in the radiological diagnosis of bowel obstructions, a CT scan has higher sensitivity and specificity than an X-ray and is better at detecting important diagnostic parameters such as the grade of occlusion and its localization.

The negative culture tests and persisting high temperature associated with a moderate increase in muscular enzymes suggested the administration of dantrolene ( $1 \mathrm{mg} / \mathrm{kg} \mathrm{IV}$ ) for 3 days and the suspension of calcium channel blockers, which led to prompt remission of fever. Intense fluid resuscitation was given as supportive therapy, with serial monitoring and correction of electrolyte disturbance. Nevertheless, in the following days oligo-anuria persisted and there was no response to fluid therapy, electrolyte correction or furosemide. Creatinine values gradually increased, resulting in renal failure with a stable CPK. The patient's relatives refused dialysis and the patient died from irreversible cardiac arrest 9 days after admission.

\section{DISCUSSION}

NMS is a rare and potentially fatal complication occurring in patients treated with antipsychotic drugs. NMS should not be confused with malignant hyperthermia $(\mathrm{MH})$ which is a rare $(1 / 50,000)$, myopathic, familial condition, mostly characterized by autosomal dominant inheritance.

The diagnosis of NMS is clinical, according to the criteria given in Table 1. The presence of an altered state of consciousness, signs of modified autonomic functionality (such as blood pressure variations, tachycardia or perspiration), leukocytosis, or increased liver enzyme levels may furtherly support the diagnosis ${ }^{[3]}$.

NMLS, characterized by a clinical picture similar to that of NMS, may occur in patients affected by Parkinson's disease (PD) as a possible reaction to a sudden withdrawal of or abrupt reduction in antiparkinsonian drugs, especially dopamine ${ }^{[4]}$. Indeed, in addition to the withdrawal of dopaminergic drugs, the occurrence of NMLS in PD patients has also been related to other conditions such as dehydration, intercurrent infection, use of cholinesterase inhibitors, a rapid switchover from bromocriptine to pergolide, constipation, and enteral nutrition, as well as to the deterioration of parkinsonian symptoms alone. Furthermore, it has been demonstrated that low levels of dopamine metabolite (homovanillic acid) in the cerebrospinal fluid may correlate with the occurrence of NMLS regardless of the dosage of levodopa ${ }^{[5]}$.

Given the apparent normal daily intake of dopaminergic drugs, our patient presented with an atypical clinical picture, which suggested specific investigation of intestinal absorption should be carried out. 
Neuroleptic malignant syndrome (NMS) diagnostic criteria

Hyperthermia $>100.4^{\circ} \mathrm{F}$ or $>38.0^{\circ} \mathrm{C}$ on at least two occasions

Rigidity

Elevated creatine phosphokinase, at least 4 times the upper limit of normal

Sympathetic nervous system lability, defined as the presence of two or more of these features:

elevated blood pressure, blood pressure fluctuation, diaphoresis or urinary incontinence, tachycardia and tachypnea

Negative for infectious, toxic, metabolic and neurological causes

Table 1: Neuroleptic malignant syndrome (NMS) diagnostic criteria

\section{CONCLUSIONS}

In the absence of modifications in drug daily administration, reduced intestinal absorption may simulate drug withdrawal. Physicians involved in the treatment of PD patients should take into consideration the possibility of dopaminergic drug malabsorption due to PB as a possible cause of NMLS, as in the case reported here. A clinical picture broadly indicative of NMLS should suggest timely investigation of bowel functionality in order to exclude malabsorption issues, thus avoiding a delay in NMLS diagnosis which could result in a fatal outcome.

\section{REFERENCES}

1. Thomas A, Onofrj M. Akinetic crisis, acute akinesia, neuroleptic malignant-like syndrome, parkinsonism-hyperpyrexia syndrome, and malignant syndrome are the same entity and are often independent of treatment withdrawal. Mov Disord 2015;20:1671; author reply 1671-1672.

2. Fiore S, et al. A neuroleptic malignant-like syndrome (NMLS) in a patient with Parkinson's disease resolved with rotigotine: a case report. Acta Biomed 2014;85:281-284.

3. Berman BD. Neuroleptic malignant syndrome: a review for neurohospitalists. Neurohospitalist 2011;1:41-47.

4. Ueda M, Hamamoto M, et al. Biochemical alterations during medication withdrawal in Parkinson's disease with and without neuroleptic malignant-like syndrome. J Neurol Neurosurg Psychiatry 2011;71:111-113.

5. Gurrera RJ, et al. An international consensus study of neuroleptic malignant syndrome diagnostic criteria using the Delphi method. J Clin Psychiatry 2011;72:1222-1228. 